\title{
APLICACIÓN FILOSÓFICA EN EL PROBLEMA DE INVESTIGACIÓN CIENTÍFICA
}

\author{
PHILOSOPHICAL APPLICATION IN THE PROBLEM OF SCIENTIFIC RESEARCH \\ Juan Guimerm o Miñano Lecaros * \\ Docente Principal de la Facultad de Ciencias Contables \\ Juan Carlos Orelano Antunez ${ }^{* *}$ \\ Docente Auxilarde ia Facultad de Ciencias Contab ies \\ Universidad Nacional Mayor de San Marcos-UNMSM / Lima-Perú \\ [Recepción: Agosto 2016/ Conformidad: Setiembre 2016]
}

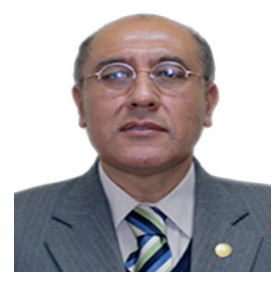

\section{RESUMEN}

Básicamente, la filosofía posee un problema que es el resolver si el origen de las cosas provienen de un hecho realizado por un ser sobrenatural y que es expresado como origen "idealista" y otro, que sostiene, que el origen de las cosas, es la evolución misma de la materia y es conocido como origen "materialista"; obviamente representado por grandes pensadores como Aristóteles, tomas de Aquino, Kant y Demócrito, Bacon y Locke entre otros y respectivamente. En tal sentido, estas dos corrientes filosóficas, opuestas en si (idealismo y materialismo), exponen las formas de conceptuar el origen de la cosa. Estas dos corrientes filosóficas, han generado una serie muy amplia de corrientes que sostienen lo expresado anteriormente; sin embargo para una conceptualización mucho más pragmática para la realización de nuestra investigación, consideramos solamente las dos corrientes filosóficas básicas. Ello permitirá poder analizar y aplicar el concepto filosófico en el desarrollo del problema de investigación y sobre todo determinar las variables que conforman el problema en el desarrollo de la investigación.

\section{Palabras Clave:}

Cosa, Materialismo, Plano, Sujeto, Conciencia, Entorno, Problema.

\begin{abstract}
Basically, the problem philosophy is to solve if whether the origin of things come from a fact realized by a supernatural being -expressed as an "idealistic" origin- or the other, which states, that the origin of things, is evolution of the matter itself and is known like "materialistic" origin. This is obviously held by great thinkers like Aristotle, Aquinas, Kant and Democritus, Bacon and Locke among others respectively. In this sense, these two opposed philosophical currents (idealism and materialism), expose the ways of conceptualizing the origin of the "thing" or object, generating a very wide series of currents that support what was previously expressed. However, for a much more pragmatic conceptualization for the realization of our research, we consider only these two basic philosophical currents. This will allow us to analyze and apply the philosophical concept in the development of the research problem and, above all, to determine the variables that make up the problem in the development of research.
\end{abstract}

\section{Keywords:}

Thing, Materialism, Level, Subject, Consciousness, Environment, Problem.

\footnotetext{
*Doctor en Ciencias Contables y Empresariales, por la UNMSM, Magíster en Auditoría, por la UNMSM, Contador Público por la UNMSM. Correo: jminanol@unmsm.edu.pe

** Master en dirección de empresas PAD UDEP, Consultor en Sistemas de gestión de la Calidad BID - SNI, Especialista en Tributación de la Universidad de Lima , Contador Público - UNMSM. Correo: jorellanoa@unmsm.edu.pe
} 


\section{INTRODUCCIÓN}

En la investigación científica, para la determinación del problema a desarrollarse, el sujeto investigador, debe asumir una posición filosófica para conceptualizar y entender la existencia del problema. Esta posición filosófica, no necesariamente es asumir una postura ideológica; es sencillamente la expresión del grado de conciencia sobre la realidad objetiva que nos circunda.

Es decir, la determinación de un problema real y existente en un entorno (ente, en el cual se desarrolla un ámbito de gestión pública o privada), refleja la posición materialista de interpretar las cosas; pues existe un alto grado de poder demostrar la existencia del mismo.

Sin embargo, cuando presentamos entornos no existentes, que han sido creados por la imaginación y no es factible su demostración de existencia, entonces estamos ante una concepción de la cosa como idealista.

De la comparación de ambas concepciones, naturalmente la primera es la de mayor conceptualización por los investigadores, porque sencillamente abordan problemas de carácter objetivo, demostrable y conceptualizado en la realidad objetiva y material.

\section{MARCO TEÓRICO}

\section{Filosofía:}

Del griego: "amor a la sabiduría”, es la ciencia sobre las leyes universales a las que se hallan subordinadas tanto el ser (la naturaleza y la sociedad), como el pensamiento del hombre, el proceso del conocimiento.

El sujeto, por la acumulación de conocimientos y el actuar reflexivo, permite apreciar el conjunto de diversas actividades sociales, económicas, educativas, etc., que lo rodea y aprecia la existencia de uno o varios problemas que está afectando el orden lógico del desarrollo de la cosa. Ello significa asumir una posición filosófica, la misma que se convierte "en método científico constituido por principios filosóficos científicos", por otro lado, también se conoce que el "principio del materialismo, que es el conocimiento de la realidad -natural, social y del pensamiento, no pueden ser penetrados, en su esencia, sin el auxilio del materialismo dialecto e histórico, cuyos principios y leyes generales hemos visto supra".

En tal sentido, el sujeto investigador, detecta problemas de investigación y los plantea a mérito de su grado de conciencia y acumulación de conocimientos; el cual para las ciencias contables, se aborda a entornos (instituciones públicas y privadas), "espacios en el cual se aprecia objetivamente la interrelación de los sujetos, que es para un modelo epistemológico, la institución o ente en el cual se desarrolla una dinámica de gestión” . Por ello, "los conocimientos de la filosofía se refieren fundamentalmente a las leyes universales que rigen tanto el ser (natural o social), como el pensamiento y el proceso del conocimiento".

\section{Ser y Observación}

Ser, filosóficamente definido como el sujeto, que es la propia evolución de la materia y poseedor de conciencia al observar la realidad objetiva y material que lo circunda, su grado cognoscitivo le permite diferenciar las categorías lógicas de lo verdadero y falso; que es la base metodológica para la determinación del problema.

La observación del contexto real, que definido como "lo objetivo, indica que las cosas, las propiedades y las relaciones existen fuera e independientemente de nosotros “

De allí, que la observación como actividad sensorial es permanente del sujeto investigador, le permite apreciar los problemas asociados a su carácter cognoscitivo que el posee, por ejemplo, en su entorno laboral, es consciente de la existencia de problemas, pero apreciara rápidamente de aquellos que se encuentra asociado a su conocimiento, tal es el caso, si es contador, tendrá la inmediata acción de poder detectar algún problema de índole contable, siempre en relación a su acumulación de conocimientos y experiencia profesional. Es por ello, que la acción de observación del sujeto, también está vinculado al grado de conciencia del mismo.

\section{Materia y Conciencia}

La materia, "como categoría filosófica, designa la 
realidad objetiva que existe con independencia de la conciencia y en esta se refleja”.

También es conceptuada, como la multiplicidad infinita de todos los fenómenos existentes, teniendo cada uno de estos fenómenos una identificación explícita, que asociado al conocimiento del sujeto, este lo percibe.

En las organizaciones públicas o privadas, los actos de gestión y el desarrollo mismo de la organización, permite constantemente, la interacción de hechos lógicos de verdadero o falso, siendo para lo segundo, la variable que refleja la existencia del problema. La conciencia, "es la forma superior propia tan solo del hombre, del reflejo de la realidad objetiva”.

El sujeto al observar y apreciar la interacción de las cosas, advierte y distingue lo bueno y lo malo, lo correcto y lo incorrecto, lo verdadero y lo falso. Por lo tanto, en un acto de conciencia, detecta un problema, analiza su evolución y situación actual y le permite presentar una alternativa de solución, el cual, estará siempre asociado a su grado de conocimiento.

El Lenguaje, como expresión del sujeto, ejerce un papel muy importante en la relación sujeto-realidad objetiva, pudiéndose reflejar como:

\section{SUJETO $\Rightarrow$ OBSERVACIÓN $\Rightarrow$ CONCIENCIA $\Rightarrow$ LENGUAJE}

La dinámica de la interrelación del sujeto y observación, se refleja porque permanentemente hay una actividad de la realidad objetiva y material; para luego desarrollarse la actividad de conciencia, como la acción que determina lo bueno y lo malo, lo verdadero y lo falso y expresarse mediante el lenguaje, la existencia de un problema a ser desarrollado para poseer su alternativa de solución. De lo expresado, se coincide con Hernández, cuando esboza los elementos para el planteamiento del problema y expresa la "evaluación de las deficiencias en el conocimiento del problema".

\section{Saber científico y anunciamiento del problema}

El saber, "producto de la actividad social del trabajo y de la acción cogitativa del sujeto, constituye la reproducción ideal, en forma de lenguaje de las conexiones sujetas a la ley objetiva del mundo" . El saber, también como acumulación de conocimientos, "es el reflejo activo y orientado de la realidad objetiva y de sus leyes en el cerebro humano. La fuente del conocimiento, lo constituye la realidad exterior que circunda al hombre y que actúa sobre el"

A mérito de la acumulación de conocimientos, la observación y el grado de conciencia, el sujeto investigador, enuncia el problema. Según Hernández, "enunciar un problema de investigación, consiste en presentar, mostrar y exponer las características o los rasgos del tema, situación o aspecto de interés que va a estudiarse, es decir, describir el estado actual de la situación del problema".

Los criterios a seguir en el planteamiento del problema, "este, debe implicar la posibilidad de realizar un prueba empírica, es decir, la factibilidad de observarse en la "realidad única y objetiva". En lo que refiere a su determinación, el problema científico "significa ubicar el problema de investigación dentro del contexto global del sistema o sub-sistema natural u social, estableciendo sus relaciones de causalidad o de correlación".

De lo expresado anteriormente, consideramos que el anunciamiento del problema, debe estar indisolublemente ligado a tres elementos que permitan su exposición: sujeto, entorno y problema; donde el sujeto como elemento racional, posee un alto grado de conciencia que unido a la acumulación de conocimientos, le permite detectar un problema existente relacionado cognoscitivamente a él, este hecho es factible, porque se desarrolla en un plano material objetivo que es el entorno, lugar en el cual interrelacionan sujetos y de allí, la existencia del problema, que en el ámbito de la gestión institucional, por ejemplo, a desarrollarse en la actividad pública o privada, refleja una determinación lógica de lo falso, debiendo ser verdadero, la variable que interrelaciona en dicho espacio de gestión .

\section{Acción reflexiva sobre el problema}

Determinado el problema de investigación, este debe ser desarrollado para conocer su "origen, evolución y situación actual del mismo", a fin de poder determinar sus alternativas de solución. Es así, que el carácter lógico de la investigación, del modo dialectico, exige que un problema, debe poseer una solución. En sí, para las ciencias contables, tratar algún problema de investigación, este debe estar asociado a un impacto socio-económico interno en 
la organización pública o privada y de igual modo a nivel de gobierno.

Explícitamente, en la elección de problemas de investigación para las administraciones públicas, estas devienen en deficiencias por ausencia normativa, inadecuada interpretación y falta de sistematización automática de sus procedimientos administrativos; por lo que en las propuestas de alternativas de solución, debe contemplarse lo anteriormente señalado.

\section{METODOLOGÍA}

La característica que refleja la población de estudio, refiere al trabajo de investigación realizado y es de carácter reflexivo y observacional. Pues como se aprecia en el siguiente punto (2. CASO REAL DE LO TRATADO), se aborda un análisis sobre una tesis doctoral, aplicando la metodología reflexiva (de lo que existe), llegando a la instrumentalización de lo observado (lo analizado).

Lo que permite expresar la realidad objetiva y material, en este caso el trabajo de investigación de la tesis doctoral denominada: "Estudio de la gestión de las entidades prestadoras de salud en el sistema privado de salud en el Perú”.

\section{Caso real de lo tratado}

Recientemente en el Vice-decanato de investigación y posgrado, de la Facultad de Ciencias Contables de la Universidad Nacional Mayor de San Marcos, se ha aprobado la tesis doctoral titulado "Estudio de la gestión de las entidades prestadoras de salud en el sistema privado de salud en el Perú", elaborado por Percy Vilchez Olivares, el cual reúne los requisitos preliminares para la investigación:

- Sujeto, el investigador vinculado a la variable independiente (el problema de la salud en el Perú)

- Entorno, la organización privada prestadora del servicio de salud a la ciudadanía

- Problema, la administración del servicio de salud bajo el sistema privado, como la variable dependiente
Y que también tiene como característica, que el sujeto investigador asuma el alto grado de conciencia por su carácter cognoscitivo respecto a la administración de la salud privada en nuestro país, ello lo materializa al exponer el problema tal como la realidad objetiva y material lo refleja. No presenta constructos de carácter subjetivo, todo lo contrario aborda el espacio demostrable en cuadros estadísticos, información contable, información financiera entre otros.

Asimismo recurre a las normas y regulaciones vinculantes, lo que permite crear el marco legal de la investigación convirtiéndolo en una información con alto grado de licitud.

Empero, el concepto filosófico lo expone en el trabajo antes mencionado en el Capítulo II Marco Teorico, 2.1 Concepción filosófica y teórica del bienestar, concepción teórica del estado de bienestar, concepción filosófica y ética de justicia sanitaria, exponiendo lo concerniente al modo de pensar ético y moral sobre la salud, no se remite a una larga exposición prolija sobre los grandes pensadores de la humanidad, que si encontramos en algunos borradores de trabajo de investigación, que luego es replanteado de acuerdo a lo anteriormente señalado.

La exposición realizada, estriba en la conceptualidad del ser y observación, producto del carácter reflexivo sobre la realidad objetiva y material, que para este caso es la gestión de las entidades prestadoras de salud en el sistema privado de salud. Es decir, no existe espacio para lo imaginable, lo ideal y lo subjetivo.

Es entonces, que la investigación contiene la estructura real de la percepción para el concepto de la exposición de las variables independiente y dependiente e ingresa al espacio sociológico, es decir, la preocupación del investigador por la existencia de dos variables y que indudablemente tiene un impacto económico-social al ciudadano.

El grado de conciencia, no solamente se presenta como expresión del investigador, pues se torna en una propuesta de alternativa de solución, lo que significa la validez de lo cognoscitivo en la determinación de las variables. 
Es por ello, que el trabajo materia del presente constructo, concluye en diez conclusiones y diez recomendaciones, todas ellas con el fin de resolver los problemas relacionados a: afiliación al sistema de salud, difusión de los derechos habientes, difusión de beneficios de los programas de salud, información de los resultados financieros y económicos, revisión de las regulaciones en los procesos de contratación de prestaciones de salud, información de los resultados de las clínicas privadas, el estado debe desarrollar programas de formalización de la economía basada en los beneficios que agreguen valor a la decisión de afiliación, se revise el nivel de siniestralidad en los convenios con las entidades privadas, revisión de los costos de farmacia y que la atención a los afiliados se base en un plan de salud que incluya beneficios y coberturas.

\section{CONCLUSIONES}

1. La posición filosófica del investigador, debe ser reflejo, de la conceptualización de la realidad objetiva y material donde se aprecia el problema de investigación.

2. La observación permanente de la realidad objetiva y material, permite al sujeto poder interrelacionar lo verdadero y falso sobre el desarrollo de las cosas.

3. La conciencia, como el alto grado de elemento sensorial del sujeto, le permite una acción sobre la existencia de un problema real y objetivo.

4. La permanente acumulación de conocimientos, por un lado, enriquece el grado de conciencia del sujeto investigador y por otro, permite detectar rápidamente problemas vinculados a su carácter cognoscitivo.

5. El problema de investigación, se encuentra vinculado a un carácter de impacto socio-económico en nuestra sociedad. Por lo tanto, su proceso de desarrollo, conlleva inexorablemente a la presentación de las alternativas de solución.

6. desarrollo, conlleva inexorablemente a la presentación de las alternativas de solución.

\section{REFERENCIAS BIBLIOGRÁFICAS}

1. BERNAL, C. (2006) Metodología de la Investigación, Colombia, Pearson Ediciones.

2. BUNGE, M. (2000) La ciencia, su método y su filosofía, Buenos Aires, Ediciones Siglo Veinte.

3. CABALlERO, A. (2014) Metodología Integral innovadora para Planes y Tesis. La metodología de como formularlo, México, CENGACE LEARNING.

4. GARCIA, F. (2014); Metodología de la Investigación. Enfoque por competencias genéricas y disciplinarias, México, LIMUSA.

5. GARCIA, D. (2009) Metodología del trabajo de investigación. Guía práctica, México, Trillas.

6. HERNANDEZ, C. (2014); Metodología de la Investigación, México, Mc Graw-Hill.

7. ÑAUPASPAITAN,H. "Metodología de la investigación científica y asesoramiento de tesis, (2009), Lima, Grafica RetaiSac.

8. ÑAUPAS, H.; MEJIA, E.; NOVOA, E.; VILLA GOMEZ, A.; (2014), Metodología de la Investigación, Cuantitativa- Cualitativa y redacción de la tesis; Colombia, Ediciones de la U.

9. QUIPUKAMAYOC, Revista de Investigación Contable (2014), Lima, Facultad de Ciencias Contables, UNMSM, Volumen 22, numero 42.

10.ROSENTAL-IUDIN (1976); Diccionario Filosófico, Buenos Aires, Editorial Universo.

11.VALARINO HERNANDEZ, E. (2012); Metodología de la Investigación, paso a paso, México, Trillas

12.VILCHEZ OLIVARES, P. (2016)"Estudio de la gestión de las entidades prestadoras de salud en el sistema privado de salud en el Perú", tesis doctoral, Facultad de Ciencias Contables, Universidad Nacional Mayor de San Marcos, Lima, Perú 